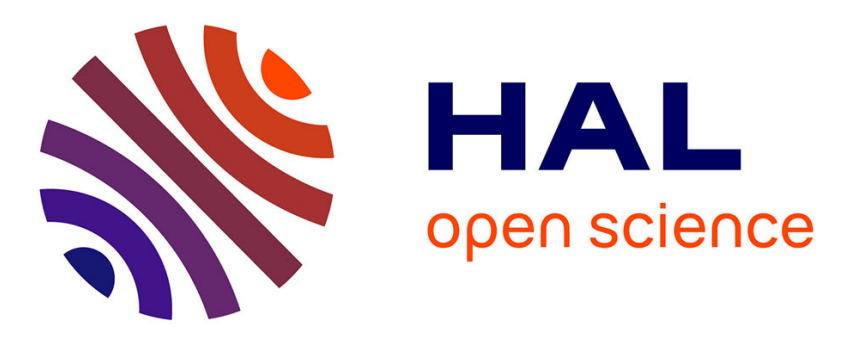

\title{
Modeling the reduction of thermal conductivity in core/shell and diameter-modulated silicon nanowires
}

Etienne Blandre, Laurent Chaput, Samy Merabia, David Lacroix, Konstantinos Termentzidis

\section{- To cite this version:}

Etienne Blandre, Laurent Chaput, Samy Merabia, David Lacroix, Konstantinos Termentzidis. Modeling the reduction of thermal conductivity in core/shell and diameter-modulated silicon nanowires. Physical Review B: Condensed Matter and Materials Physics (1998-2015), 2015, 91 (11), pp.115404 115404. 10.1103/PhysRevB.91.115404 . hal-01486117

\section{HAL Id: hal-01486117 \\ https://hal.univ-lorraine.fr/hal-01486117}

Submitted on 9 Mar 2017

HAL is a multi-disciplinary open access archive for the deposit and dissemination of scientific research documents, whether they are published or not. The documents may come from teaching and research institutions in France or abroad, or from public or private research centers.
L'archive ouverte pluridisciplinaire HAL, est destinée au dépôt et à la diffusion de documents scientifiques de niveau recherche, publiés ou non, émanant des établissements d'enseignement et de recherche français ou étrangers, des laboratoires publics ou privés. 


\title{
Modeling the reduction of thermal conductivity in core/shell and diameter-modulated silicon nanowires
}

\author{
Etienne Blandre, ${ }^{1,2}$ Laurent Chaput, ${ }^{3}$ Samy Merabia, ${ }^{4}$ David Lacroix, ${ }^{1}$ and Konstantinos Termentzidis ${ }^{1, *}$ \\ ${ }^{1}$ Université de Lorraine, LEMTA, Centre National de la Recherche Scientifique, Unités Mixtes de Recherche 7563, \\ BP 70239, 54506 Vandouvre Cedex, France \\ ${ }^{2}$ INSA, CETHIL, Centre National de la Recherche Scientifique, Unités Mixtes de Recherche 5008, 69621 Villeurbanne, France \\ ${ }^{3}$ Université de Lorraine, Institut Jean Lamour, Centre National de la Recherche Scientifique, Unités Mixtes de Recherche 7198 , \\ Boulevard des Aiguillettes, BP 23, 54506 Vandouvre Les Nancy Cedex, France \\ ${ }^{4}$ Université de Lyon 1, ILM, Centre National de la Recherche Scientifique, Unités Mixtes de Recherche 5306, 69621 Villeurbanne, France
}

(Received 7 November 2014; revised manuscript received 13 February 2015; published 3 March 2015)

\begin{abstract}
The thermal conductivity of a series of structural, diameter, and core/shell modulated silicon nanowires is computed by molecular dynamics simulations using the nonequilibrium method. The purpose of this study is to appraise the impact of surface roughness, amorphous parts integration, and diameter modulation on the thermal conductivity reduction. The addition of both amorphous regions and nanoconstrictions allows us to reach a thermal conductivity close to that of the amorphous pristine nanowires, while preserving a certain degree of crystallinity. This could result in interesting properties for thermoelectric applications.
\end{abstract}

DOI: 10.1103/PhysRevB.91.115404

PACS number(s): 65.80.-g, 31.15.xv, 63.22.Kn

\section{INTRODUCTION}

Finding innovative thermoelectric materials is the object of intense research today. There are several challenges to address like the efficiency, the sustainability, and the processibility of these materials. However, silicon based nanostructures offer alternative routes, with the advantages of good engineering and low production cost, which will both be important in the near future for large scale applications for thermoelectric technologies. In this respect, to balance the weak efficiency of bulk material properties of silicon compared to other thermoelectric materials, innovative concepts and devices are important. Due to the well-controlled production processes, those are possible for silicon, but unfortunately not for other thermoelectric materials. Such possibilities are investigated in the present work through the use of nanostructuration.

In general, nanostructured materials have a much lower thermal conductivity than bulk materials, due to phonon confinement and boundary scattering, especially in semiconductor nanowires (NWs) [1,2], leading to a higher figure of merit:

$$
Z T=\frac{S^{2} \sigma T}{K} .
$$

Here $S$ is the Seebeck coefficient, $\sigma$ is the electrical conductivity, and $K$ is the thermal conductivity (TC) of the material which includes both the lattice and the electronic contributions. To further improve this figure of merit, it is often accepted that the more efficient approach is to reduce the TC. To reach this objective, a series of geometrical and structural parameters of nanowires such as (i) size, (ii) surface structure, (iii) structural composition, and (iv) amorphization may be tuned by choosing different growth protocols and conditions. All of those modifications affect the transport properties [3]. For example, the decrease of the Si nanowires cross section leads to a $Z T$ close to 1 , which is already a rather large value compared to most bulk materials. To increase $Z T$ beyond

*konstantinos.termentzidis@univ-lorraine.fr this value, new nanostructured materials must be designed. Surface roughness of the nanowires can also significantly increase the figure of merit [4,5]. Following this idea, an engineered surface roughness, as a diameter modulation of the nanowires, can also control the decrease of the thermal conductivity $[6,7]$. Such cross-section modulated nanowires are interesting candidates for thermoelectric applications $[8,9]$, and during the last two to three years an increasing interest appeared in the literature $[7,10,11]$. This class of nanowires has already been achieved [12,13]. On the other hand, the addition of amorphous regions around or inside the latter ones can also have an important impact on their TC. This has been shown in a couple of studies for the case of crystalline-core/amorphous-shell NWs [3,14-16].

In this paper, we explore how the combination of nanostructuration and partial amorphization may be used to reduce the conductivity of silicon nanowires. To the best of our knowledge, this is the first illustration of a systematic study of several parameters combining the two effects. Furthermore, we have studied the thermal properties of a new class of nanowires, made of an amorphous core and a crystalline shell ( $a c / c s$ NWs). The fabrication of the $a c / c s$ NWs has been reported very recently in the case of $\mathrm{Ge}_{2} \mathrm{Sb}_{2} \mathrm{Te}_{5}$ core/shell nanowires [17] and also has been observed by V. Lysenko in small scale during heavy-ion bombardment of a forest of crystalline silicon nanowires [18,19]. We address our theoretical study through molecular dynamics (MD) simulations. The latter technique has been successfully applied in recent years to the study of thermal transport across semiconductor nanowires $[3,6,20]$. Besides, it has the advantage to provide information on the local vibrational properties of the nanowire.

The paper is structured as follows: in Sec. II we detail the MD methodology and the modeled structures. Section III is divided in three subsections that address each kind of studied nanowires. We first consider the impact of the diameter modulation on the TC of pristine crystalline silicon nanowires and we investigate the influence of the periodicity of the modulation. In the second subsection, the impact on the TC of an amorphous phase in constant cross-section core/shell 
silicon nanowires is evaluated, by varying the amorphous phase fraction. In this subsection, two types of core/shell nanowires are presented: (i) the most common type of $a-S i / c-S i$ nanowire, which is the crystalline-core/amorphousshell $(c c / a s)$ and which can be realized through the interaction of a crystalline structure with the oxygen or caused by ion irradiation [18], and (ii) amorphous-core/crystalline-shell nanowires $(a c / c s)$, which can be elaborated by a swift and spatially resolved heavy-ion bombardment [18]. Finally, in the third subsection we report the TC of $c c / a s$, which are also diameter-modulated silicon nanowires for three different configurations. Then, a summary and perspectives to this work are given in the concluding paragraph.

\section{MOLECULAR DYNAMICS}

\section{A. Nonequilibrium molecular dynamics method and simulation setup}

We used the nonequilibrium molecular dynamics method [21,22] to compute the TC of modulated silicon nanowires. For such simulations, when steady state is reached, the temperature profile along the nanowire length and the heat flux through the structure are extracted. Assuming diffusive heat transport, which is the case here due to strong phonon scattering in nanowires, and applying Fourier's law, one can calculate the TC [22-27]. For each modeled structure, the TC of four nanowires with different lengths was calculated in order to extrapolate the TC corresponding to an infinite length [28], while the phonon mean free path has been estimated with the equation $1 / l_{\mathrm{eff}}=1 / l_{\infty}+4 / L_{z}$ [23], where $L_{z}$ is the length of the nanowire from the hot to the cold thermostat and $l_{\text {eff }}$ is the phonon mean free path. This estimation provides the average phonon mean free path, which is the mean free path of energy modes that carry heat and contribute to the thermal conductivity. The MD code LAMMPS [29,30] is used for our calculations.

In what concerns boundary conditions, periodic ones are set in the $z$ direction, which is the main axis of the nanowires, while in the $x$ and $y$ directions we use free boundary conditions [20]. At the extremities of the nanowires, fixed atoms within regions of 1-nm width are set in order to avoid any movement of the nanostructures. Alongside the fixed atom regions, we set the hot and cold thermostats, with length of the thermostats $1.086 \mathrm{~nm}\left(2 \alpha_{0}\right)$. Thermostating is performed through rescaling of the velocities of the atoms belonging to the group of the heat sink and heat source, for each time step. They induce a heat flux within the system. For all simulations the mean temperature was $300 \mathrm{~K}$, while the heat source was at $330 \mathrm{~K}$, and the heat sink was at $270 \mathrm{~K}$. The time step used is $5 \mathrm{fs}$. The nanowire lengths vary from 13.3 to $49.3 \mathrm{~nm}$. The classical Stillinger-Weber potential for the pure crystalline system is used [31], while its modified version, the so-called SW-VBM [32], is employed for all $a-S i / c-S i$ nanowires. In Fig. 1, a characteristic temperature profile of a diameter-modulated crystalline-core/amorphous-shell silicon nanowire is displayed. The atoms are colored by their mean temperature for a simulation run with a duration of $5 \mathrm{ps}$, which is enough here to obtain a linear temperature profile.

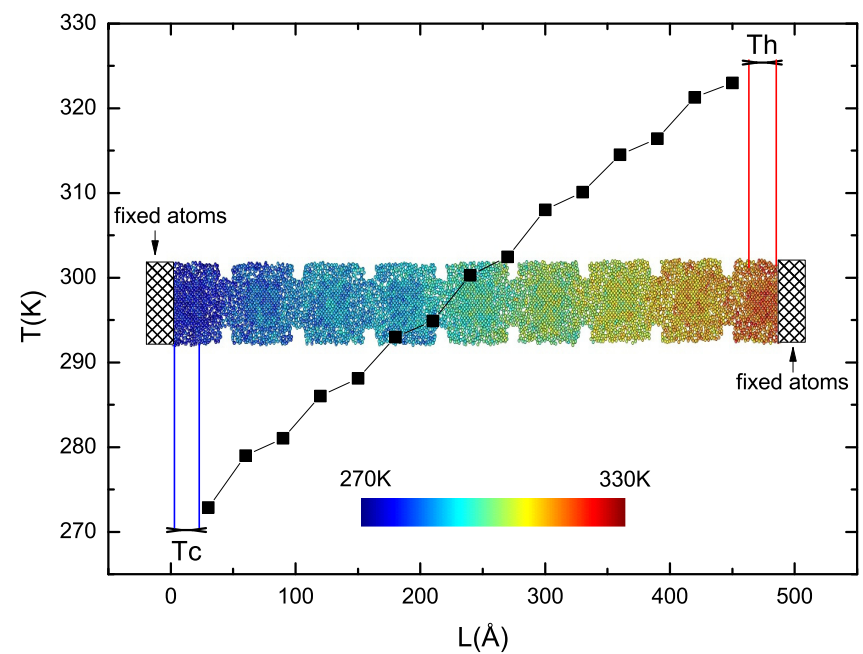

FIG. 1. (Color online) Temperature profile of a diametermodulated nanowire with crystalline nanoinclusions. The atoms are colored by their mean temperature. The temperature profile exhibits different slopes corresponding to the diameter modulation effect on TC.

\section{B. Modeling of core/shell nanowires}

In the present work an important issue is to build realistic amorphous/crystalline structures. Here, we used the method presented in previous papers [33,34] and that has been already applied in the case of amorphous/crystalline superlattices [35] to determine the atom location in the nanostructures. This technique is achieved in two main stages which involve several steps. The first stage is the creation of a realistic silicon amorphous slab and the starting step is to define a random distribution of atoms inside a simulation box with the desired dimensions. Then, the system is annealed using a Nose-Hoover thermostat [36] at $2400 \mathrm{~K}$, during $50 \mathrm{ps}$ in the $N-V-T$ ensemble. The next step is the quenching of the system, with a rate of $10^{12} \mathrm{~K} \mathrm{~s}^{-1}$. Finally, a global relaxation in the isothermal isobaric ensemble $(N-P-T)$ is performed. It is important to notice at this point that the potential used for this "first stage" is the modified Stillinger-Weber potential (SW-VBM) for amorphous silicon [32]. It allows us to obtain a realistic amorphous phase. Once the slab is generated, the quality of the amorphous phase is checked using the radial distribution function, which is compared with $\mathrm{X}$-ray data [37,38], the coordination number [38], the bond angle, and the bond length distribution [34]. We have checked the reproducibility of the amorphous phase, creating several amorphous slabs. They each have the same radial and bond distribution function, as well as coordination number. Thus, all the following nanowires which contain amorphous phase are fabricated with the same amorphous slab as the starting structure, assuming that no significant modifications of the thermal and vibrational properties can arise.

In the second stage of the amorphous/crystalline nanostructure elaborations, certain regions including several atoms are removed in order to create empty spaces within the amorphous slab. These empty spaces are then filled with the crystalline phase. In the following step, the system is annealed at $300 \mathrm{~K}$ to obtain a pressure near $0 \mathrm{GPa}$, and finally the energy of the 


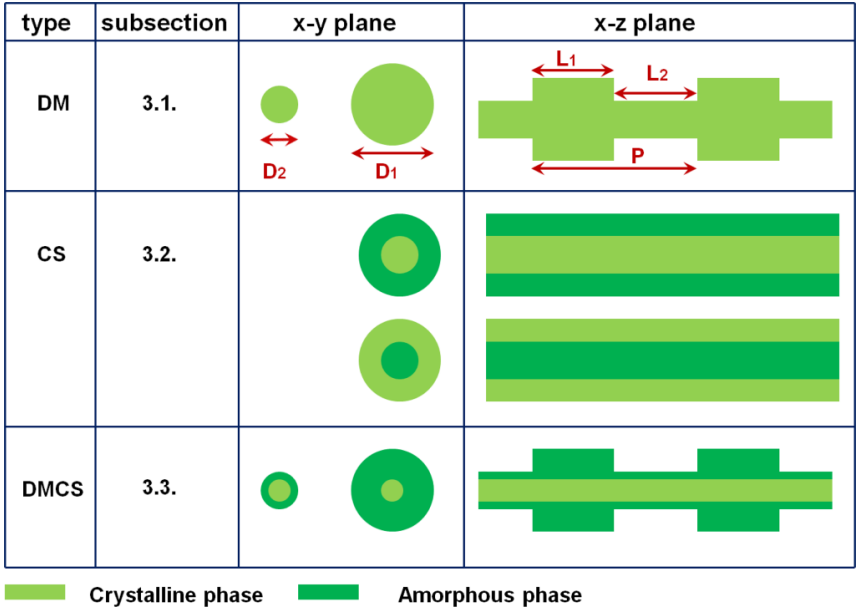

FIG. 2. (Color online) Schematic representation of the diametermodulated crystalline silicon nanowires (DM), the $a-S i / c-S i$ core/shell (CS) nanowires, and the $a-S i / c-S i$ diameter-modulated core/shell (DMCS) nanowires.

system is minimized. Once again, at the end of this second stage we ensure that the build structure is correct. To do this, the quality of the interfaces in amorphous/crystalline structures is controlled with the atomic energy profile along the radial axis of the nanowires, starting from the center and going to the external radius [34]. The calculated interfacial energy $0.46 \mathrm{~J} \mathrm{~m}^{-2}$ [34] is compared with tight-binding results $0.49 \mathrm{~J} \mathrm{~m}^{-2}$ [39] and experimental ones $0.48 \mathrm{~J} \mathrm{~m}^{-2}$ [40], leading to an excellent agreement.

As it was mentioned in the introduction, we study three types of nanowires with different morphologies (see Fig. 2). The first ones are pristine crystalline nanowires with diameter modulation (labeled DM). The second class is of the core/shell type nanowires (labeled CS), with constant diameter. Among them there are two subtypes: the $c c / a s$ and the $a c / c s$. The considered external diameter for these nanowires is $6 \mathrm{~nm}$ and for this case nanowires with several ratios of the crystalline/amorphous phases are created by varying the core radius. Finally there are the diameter-modulated $c c / a s$ nanowires, where three distinctive configurations are considered (labeled DMCS, Fig. 2): (i) the $C 1$ configuration, in which the diameter of the crystalline core remains constant and where only the amorphous shell diameter is modulated; (ii) the $C 2$ configuration, in which both the core and the shell diameters are modulated; and (iii) the $C 3$ configuration, consisting of crystalline nanoinclusions inside a diametermodulated amorphous shell.

The CS and DMCS nanowires were constructed with the method described above for the amorphous phase integration. We modeled all types of nanowires with a circular cross section.

\section{THERMAL CONDUCTIVITY OF SILICON NANOWIRES}

\section{A. Diameter-modulated crystalline silicon nanowires (DM)}

The size reduction and the surface roughness of nanowires have been reported as major parameters to reduce their

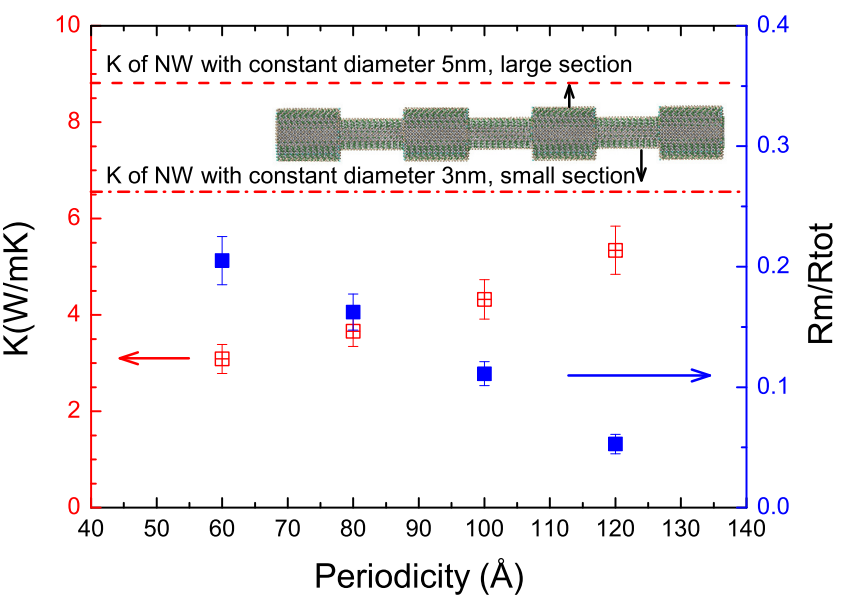

FIG. 3. (Color online) Evolution of TC and of the ratio $R_{m} / R_{\mathrm{tot}}$ as a function of the periodicity $P$ of the diameter modulations.

TC $[5,41]$. A recent work on diameter and lattice modulated $\mathrm{SiC}$ nanowires [6] showed an important decrease of the thermal conductivity compared to the one of nanowires with a constant diameter. In this section we investigate the impact of the diameter modulation of crystalline silicon nanowires on the TC. The free parameter in our study is the periodicity $P$ of diameter modulation in the direction of the nanowires axis (see Fig. 2). The latter one is in the range $60<P<120 \AA$. For these nanowires, the small radius is $D_{2}=3 \mathrm{~nm}$, and the large radius is $D_{1}=5 \mathrm{~nm}$ and the corresponding lengths are $L_{1}=L_{2}=P / 2$ (Fig. 2 top).

For all periodicities, we report in Fig. 3 the calculated TC of nanowires extrapolated to infinite size [28]. We give also the values of the thermal conductivity of a constant diameter silicon nanowire with 3- and 5-nm diameter for the sake of comparison. The temperature jump across the constrictions is computed, and we report the thermal constriction resistance [6], defined as $R_{m}=\frac{\Delta T}{\varphi}$, where $\Delta T$ is the temperature jump at the interface, and $\varphi$ is the heat flux through the structure. $K$ and the ratio $R_{m} / R_{\text {tot }}$ are plotted in Fig. 3, where $R_{\mathrm{tot}}$ is the total thermal resistance of the whole structure. Our results show the same tendencies as those previously reported for such nanowires [6,10]. The diameter modulation allows us to reach a lower TC than the one of the smaller constant diameter nanowires due to the additional scattering mechanism from the constrictions which behave as scattering centers. The qualitative results in this study are owing to the influence of the periodicity of the diameter modulation. When the periodicity decreases the TC decreases, even by a factor of 2. Decreasing the periodicity is equivalent to increasing the constriction concentration $x$, and therefore the scattering probability of heat carrying phonons, or inverse relaxation time $1 / \tau$. Indeed the linear behavior observed in Fig. 3 suggests that $1 / \tau \propto x$, as for point scattering. The ratio $R_{m} / R_{\text {tot }}$ shows a clear decrease as $P$ is increased. This is however not surprising due to the change in the interface/volume ratio as $P$ is increased since $R_{m}$ is calculated in a fixed volume. The value of $R_{m}$ itself does not change with the periodicity and it is equal to $3.3 \times 10^{10} \mathrm{~m}^{2} \mathrm{~K} / \mathrm{W}$. This confirms our suggestion 


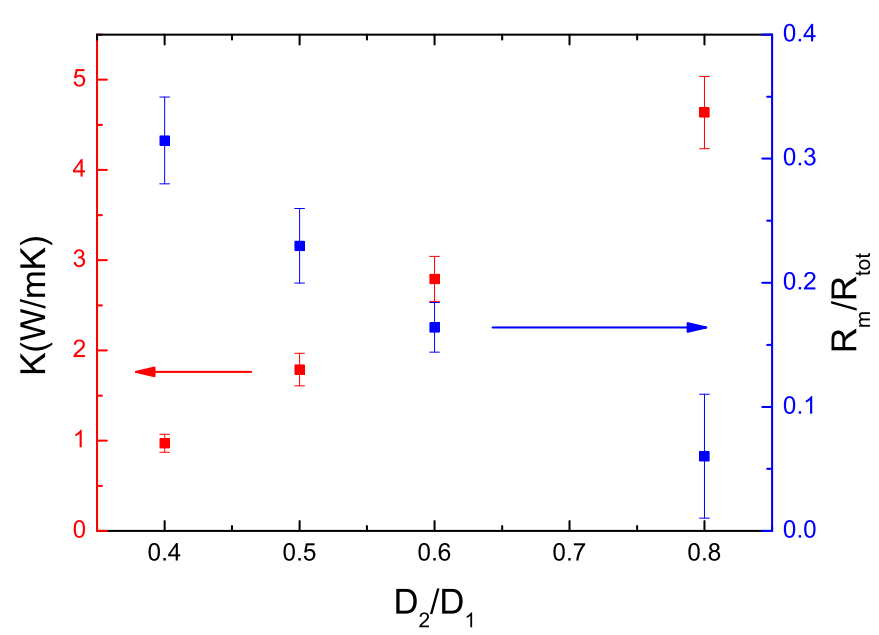

FIG. 4. (Color online) TC and thermal constriction resistance ratio $R_{m} / R_{\text {tot }}$ of single nanoconstriction as a function of the diameters ratio $D_{2} / D_{1}$.

to consider the constrictions as independent scattering centers, not interacting with each other in that range of periodicity.

To get complementary information about the behavior of the thermal conductivity we have studied a single nanoconstriction, which we have seen can be considered as an independent scattering center. It was modeled setting a thin diameter nanowire between two "bulklike" reservoirs. The latter ones have a constant diameter $D_{1}=5 \mathrm{~nm}$ and constant length $L_{1}=$ $10 \mathrm{~nm}$. The characteristic lengths of the "thin nanowire" are its constant length $L_{2}=5 \mathrm{~nm}$ and its diameter $D_{2}$ which can vary from 2 to $4 \mathrm{~nm}$. Both nanowire and reservoirs have a circular cross section. Here, the size of the reservoirs is limited because periodic boundary conditions cannot be simply applied to a part of this cylindrical nanostructure. Thus, increasing the number of atoms in the reservoir drastically increases the computational time to reach convergence. Besides, we must also check that the thermostats, set in reservoirs, are far enough from the nanoconstriction. By varying the nanowire diameter $D_{2}$, we succeed to vary the ratio $D_{2} / D_{1}$ and finally study the effect of the height of a nanoconstriction on the TC and the thermal constriction resistance ratio $R_{m} / R_{\mathrm{tot}}$. These results are depicted in Fig. 4.

It can be noted that the thermal constriction resistance ratio decreases significantly when the $D_{2} / D_{1}$ ratio increases. This means that the more $D_{1}$ and $D_{2}$ are different the smaller the thermal conductivity will be. Our results are consistent with those obtained by $\mathrm{He}$ and Galli [16], showing that increasing the depth of the ripples in rough nanowires lowers the $\mathrm{TC}$ of the nanostructure. In their work this behavior was attributed to an increase of the free surface scattering.

To further understand the resistive effect of the modulations which are material dependent, we compute the VDOS (see Fig. 5) which is obtained through a Fourier transform of the time velocity correlation functions $[42,43]$. The VMD module [44] was used to compute both the autocorrelation and Fourier's transforms. Specific regions of the system have been chosen, with widths of $4 \AA$, which are localized at each side of the nanoconstriction. The names for these regions are defined in Fig. 6.

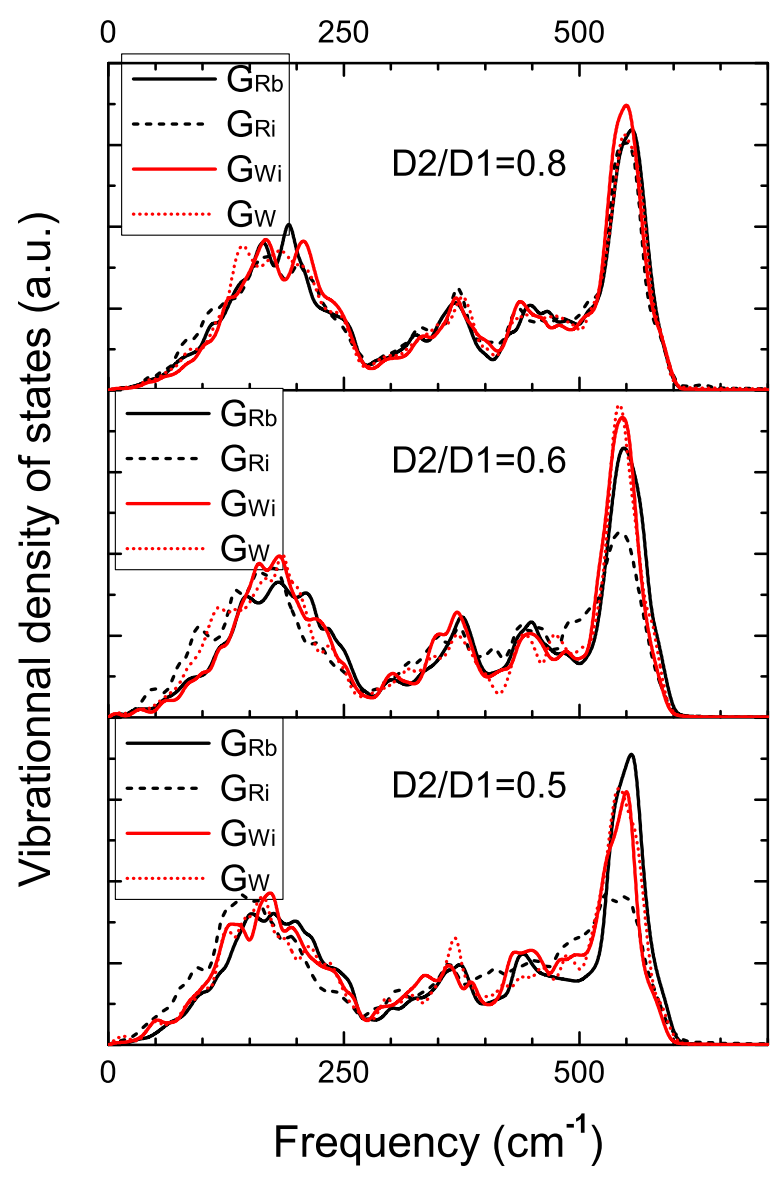

FIG. 5. (Color online) VDOS inside the four regions $G_{R b}, G_{R i}$, $G_{W i}$, and $G_{W}$, for three diameter ratios of the nanowires $\left(D_{1} / D_{2}=\right.$ $\left.0.8 ; D_{1} / D_{2}=0.6 ; D_{1} / D_{2}=0.5\right)$, using the SW interatomic potential [31].

The VDOS of the group of atoms far from the nanoconstriction $\left(G_{R b}\right)$ is represented with a black line in all three panels. This is essentially the VDOS of bulk silicon. The black dashed lines correspond to the VDOS of a group of atoms in the reservoir but close to the nanoconstriction $\left(G_{R i}\right)$. The three subfigures have been obtained for three different ratios of $D_{2} / D_{1}$. One can observe a systematic decrease of the main peak of the $G_{R i}$ as $D_{2} / D_{1}$ decreases for the three configurations. The latter one corresponds to the transverse optical mode (TO) at frequency $550 \mathrm{~cm}^{-1}$. One can also observe a softening of the acoustic phonons, say those with a

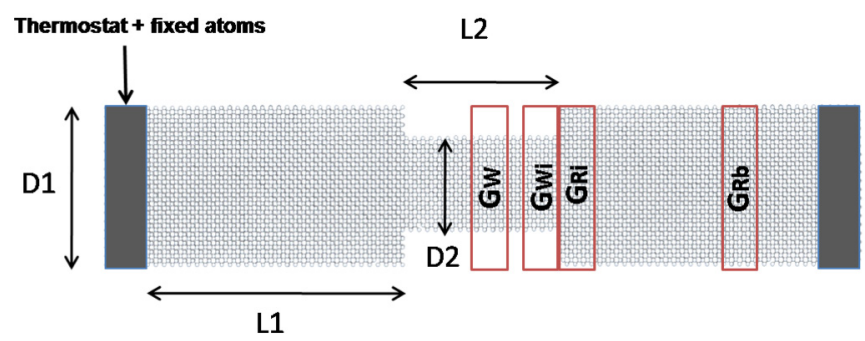

FIG. 6. (Color online) Schematic representation of a crystalline single nanoconstriction and its characteristic lengths. The red rectangles represent the groups of atoms used to extract the VDOS. 
frequency below $200 \mathrm{~cm}^{-1}$. Considering that these phonons are those which carry more heat, we can understand that this softening is another mechanism that will contribute to thermal conductivity reduction, in addition to the direct effect on scattering provided by the geometry of the nanoconstriction.

Let us focus now on the VDOS of the other two groups $(G w)$ and $(G w i)$ (Fig. 5) which are defined in Fig. 6. These atoms are located in the thin nanowire, far and close to the constriction, respectively. One can also observe a softening of the phonon frequencies, but it is now less meaningful than in the nanowire with the large diameter. Nevertheless, it is now clear that phonon softening should be included for a qualitative description of the thermal conductivity reduction in diameter-modulated nanowires. Besides, it has been observed that even small scale phonon softening can be attributed to the reduction of the thermal conductivity in a variety of materials [45-47]. The phonon softening can originate from the existence of the free surfaces, the atoms close to the amorphous/crystalline interface, the confined structures, and the roughness when it is included in modeling. All of these structural modifications induce additional scattering, which affects the phonon propagation.

\section{B. Core/shell silicon nanowires with crystalline and amorphous phases and constant diameter $(\mathrm{CS})$}

In this section the TC and the VDOS of two types of core/shell nanowires are given: (i) first for the usual crystalline core and amorphous shell ( $c c / a s)$ nanowire and (ii) second for an amorphous core and crystalline shell $(a c / c s)$. The external diameter of both types of nanowires is kept constant with a diameter equal to $D_{2}=6 \mathrm{~nm}$. We modified the core nanowire radius (internal interface) to study the impact of the amorphous fraction to the crystalline phase in both configurations. In Fig. 7, the TC of the two types of core/shell nanowires is given as a function of the amorphous fraction to the total amorphous and crystalline phases. Results for the TC of pristine crystalline $(0 \%)$ and pristine amorphous $(100 \%)$ nanowires are also given for the sake of comparison.

As intuitively expected in all cases the thermal conductivity decreases with the increase of amorphous content. The discrepancies between our study and the results obtained by Sansoz [15] are small and in absolute values should be related to the differences existing on the diameter of the cross sections ( $D_{2}=6 \mathrm{~nm}$ in our case and $D_{2}=9.8 \mathrm{~nm}$ in Sansoz's work), the shape of the cross sections (circular in our case and hexagonal in Sansoz's work), and probably the interatomic potential (SW-VBM in our study and not specified in Sansoz's case).

Concerning the $c c / a c$ system, other groups have carried out similar studies [3,14]. They also calculate the TC of such nanostructures. However, we believe that the use of the Tersoff potential instead of the SW-VBM (like in our case) and the very small cross sections, that they have considered, cannot correctly reproduce the TC of the studied nanowires. In [14], they reported a decrease of the TC by a factor of 20 , when they add an amorphous shell around a crystalline core, compared to the same size crystalline nanowire. It seems large, and we have to mention that the chosen method to prepare the amorphous/crystalline interfaces is especially crucial for

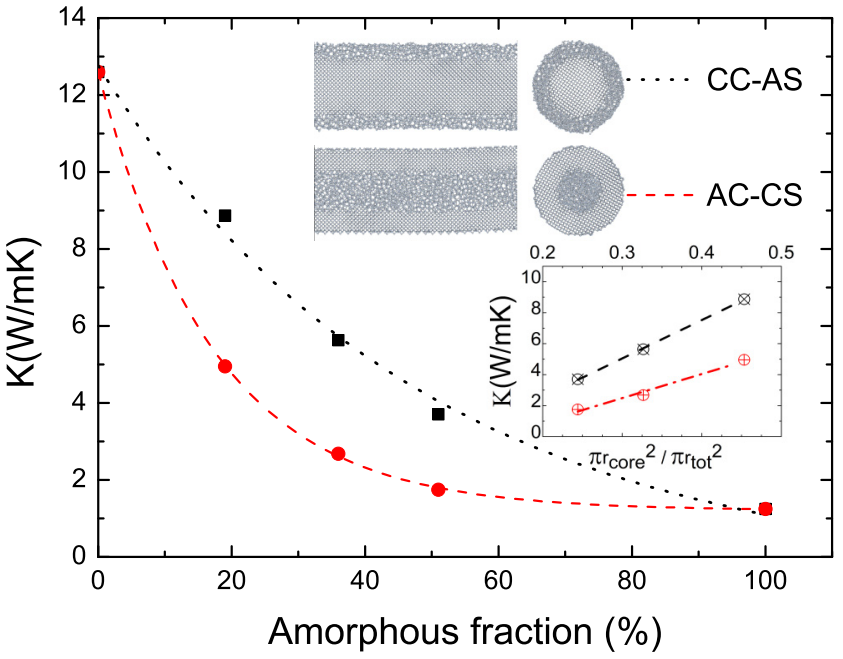

FIG. 7. (Color online) Evolution of the TC of the two types of core/shell amorphous/crystalline silicon nanowires as a function of the amorphous phase fraction (the dashed and dotted lines serve as a guide for the eye). The TCs of the pristine crystalline $(0 \%)$ and the pristine amorphous (100\%) silicon nanowires, with the same cross section, are also given. Inset: The thermal conductivity for the two types of nanowires as a function of the ratio of the cross-section area of the core and the total cross-section area (the dashed lines here are linear fittings of the data).

the assessment of transport properties. The melting/quenching technique combined with an inadequate potential for amorphous silicon can create large voids and mechanical stresses at the interfaces. This will affect the resulting TC. Our methodology to generate amorphous/crystalline interfaces takes into account experimental results as the interfacial energy or the radial distribution function to ensure the quality of both the amorphous phase and the amorphous/crystalline interface at an atomic scale [34]. We believe that the discrepancies between the different studies are first due to the way the core/shell nanowires are modeled and second due to the potential which is used for MD simulations. The latter one plays an important role for the building of the amorphous slab and it is known that the Tersoff potential has a tendency to induce a higher coordination number than the SW or the SW-VBM potentials [34].

Concerning the new class of core/shell nanowires, the one with a crystalline shell and amorphous core $(a c / c s)$, we show that it exhibits very interesting properties. First of all, the impact of the amorphous phase fraction on the overall TC follows the same tendency as before; when increasing the amorphous fraction the TC decreases. On the other hand, the TC of the $a c / c s$ nanowires is half compared to the $c c / a s$ for the same fraction of the amorphous phase. This might be exploited further for applications in thermoelectric devices. The reduced TC can be rationalized if one assumes that the thermal transport occurs mainly at the crystalline part. In that case the scattering probability of the phonons in crystalline regions, and therefore the thermal resistivity, will be proportional to the surface scattering area. The surface area is larger in the $a c / c s$ system due to the presence of both external and internal boundaries, which would explain the lower thermal conductivity. 
The inset in Fig. 7 shows the linear relation between the TCs for both types of nanowires and the ratio of the cross-section areas of the core and the whole nanowire. We can conclude that there is a geometric parameter that scales the TC when the core cross section varies. We also tried to interpret the drop of the conductivity of core/shell nanowires using a Monte Carlo approach, where phonons were allowed to propagate and be scattered by boundaries. It turned out that this simple model gave a thermal conductivity that decreases linearly with the amorphous volume fraction, and which does not depend on the position of the amorphous region (if it is at the core or at the shell). This suggests that indeed energy transfer in amorphous silicon cannot be described by collective propagative excitations, and the peculiar behavior outlined by the molecular dynamics simulations can be ascribed to the diffuse nature of the energy carriers in amorphous regions.

To get a more microscopic picture we also studied the VDOS for the $c c / a s$ and $a c / c s$ nanowires, separating the contributions of the crystalline and the amorphous regions. Results are presented in Fig. 8. For what concerns the optical peak the contribution of the amorphous phase to the VDOS is similar in both configurations. For the crystalline phase, when it is in the center of the nanowire, then it exhibits a large peak around $500 \mathrm{~cm}^{-1}$ characteristic of the crystalline behavior. This is no longer true when the crystalline phase is in the outer position (shell). In the latter case the surface

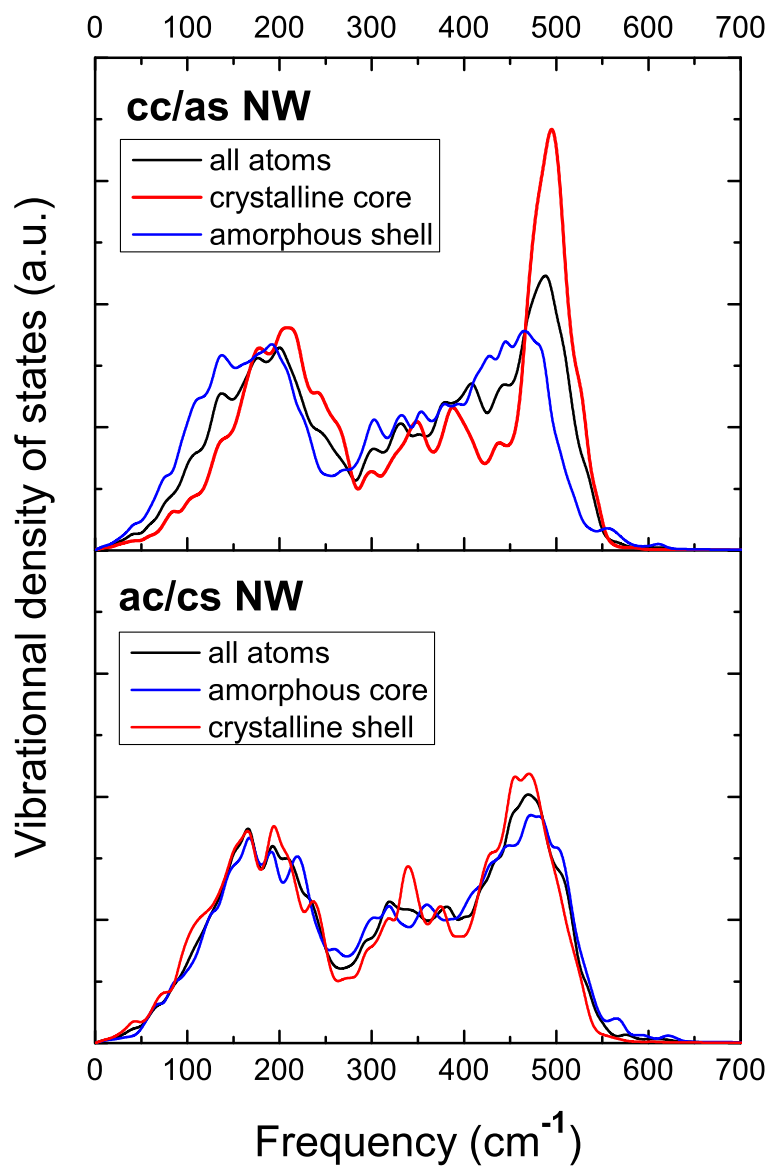

FIG. 8. (Color online) VDOS in $c c / a s$ and $a c / c s$ nanowires, using the interatomic potential SW-VBM [32]. reconstruction modifies significantly the vibrational properties and the overall behavior is close to the amorphous phase. For the acoustic modes the behavior is different. In the $c c / a s$ system the VDOSs of the amorphous and crystalline regions are rather similar to the ones of bulk systems. There is a softening of the modes in the amorphous phase compared to the crystalline one. However in the $a c / c s$ case the VDOS is shifted to an averaged intermediate value, rather similar in the crystalline and amorphous regions. Again we expect this behavior to be a consequence of surface reconstruction, which in turn contributes to reduce the thermal conductivity.

To confirm this behavior on the phonon eigenmodes, which are responsible for the transport properties rather than the distribution of phonon frequencies, we have also computed the participation ratio. It is a measure of the number of atoms participating to a given eigenmode $\alpha$ Eq. (2). It is defined by

$$
P_{\alpha}=\frac{1}{N} \frac{\left(\sum_{I=1}^{N}\left|u_{\alpha}^{I}\right|^{2}\right)^{2}}{\sum_{I=1}^{N}\left|u_{\alpha}^{I}\right|^{4}}
$$

if the $N$ atoms are labeled by $I$ and $u_{\alpha}^{I}$ is the eigendisplacement for mode $\alpha$. If $P$ atoms are participating equally to $u_{\alpha}^{I}$, then $P_{\alpha}=P / N$. Now if we call $\rho_{I}(\omega)$ the projected density of states on atom $I$ we can also define the function

$$
p(\omega)=\frac{1}{N} \frac{\left[\sum_{I=1}^{N} \rho_{I}(\omega)\right]^{2}}{\sum_{I=1}^{N} \rho_{I}(\omega)^{2}}
$$

and we have the result that at the phonon eigenfrequency $\omega_{\alpha}$, $p\left(\omega_{\alpha}\right)=P_{\alpha}$ Eq. (3).

In the following we prefer to plot $p(\omega)$ rather than $P_{\alpha}$ since the local densities of states $\rho_{I}(\omega)$ are usually available from the output of molecular dynamics calculations, whereas the phonon eigenmodes $\left(u_{\alpha}^{I}\right)$ are not. The results are presented in Fig. 9. As expected in the optical region the amorphous phases are very similar in all configurations. In the acoustic part of the spectrum the $c c / a s$ and the bulk phases are very similar, the eigenstates being more localized in the amorphous phase. It is however interesting to remark that what has been said for the frequency distribution remains true for the localization of the eigenstates: in the $a c / c s$ they are averaged to a value intermediate between the crystalline and the amorphous values of the bulk phases, and the core and shell parts of the nanowire have quite similar properties. This reveals that the phonon eigenvectors are fundamentally different in the $a c / c s$ due to surface reconstruction, which explains the drastic reduction of the thermal conductivity observed in Fig. 7. Strictly speaking there is no facet, when there is an amorphous phase at the shell of the nanowires. In the case where the crystalline phase is at the outside, the reconstruction mainly follows the (100) and the (110) facets and between them the (520) facets.

To conclude this section, that includes the most important results in the sense of a new class of nanowires, we should mention that there is a series of reasons to explain the difference in the thermal conductivity between the $c c / a s$ and the $a c / c s$ NWs. First of all phonons that carry heat (mainly in the crystalline phase) in the $a c / c s$ configuration are scattered both at the interface between the amorphous core and the crystalline shell and at the free surface that has been reconstructed. In the classical case nanowires $(c c / a s)$ there was only the internal interface as scattering center. Second, 


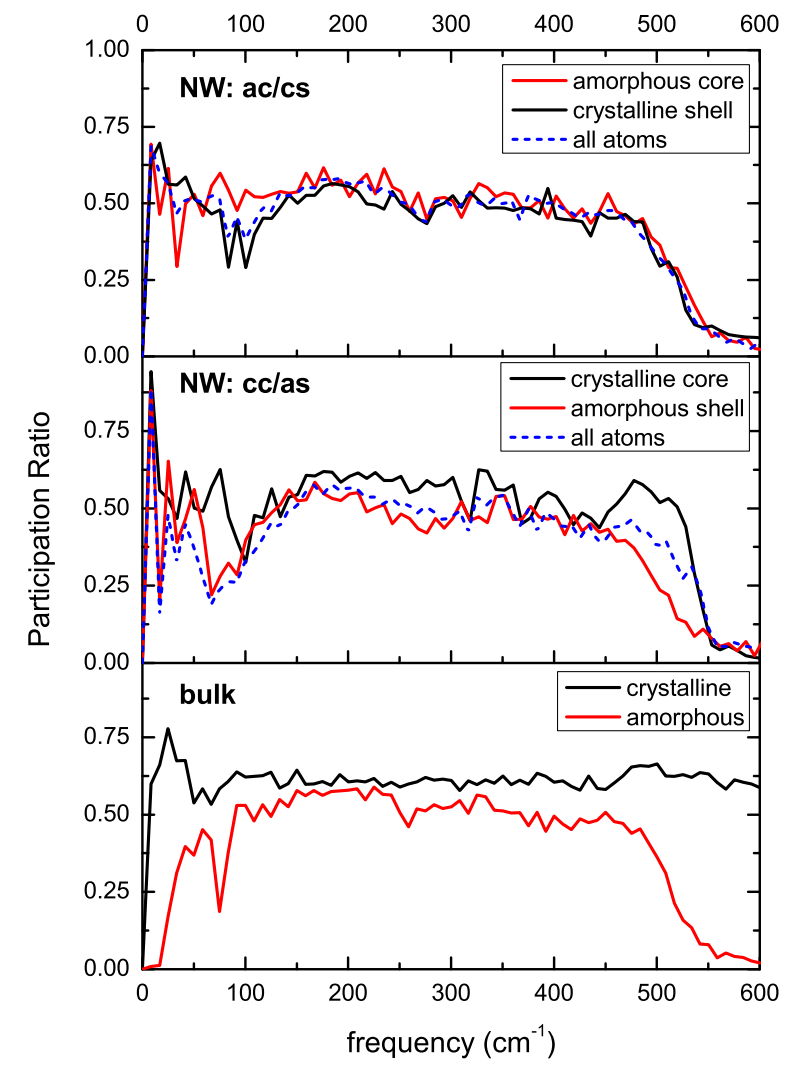

FIG. 9. (Color online) Participation ratios in the $c c / a s$ and $a c / c s$ configurations as well as in the bulk.

we calculated the phonon mean free path in the classical case to be $5 \mathrm{~nm}$, while in the $a c / c s$ NWs version it is divided by a factor of 2: $2.7 \mathrm{~nm}$. These results are for the same amorphous phase fraction in the two cases, corresponding to $19 \%$. Moreover, with the help of the VDOS, we can see that in the $a c / c s$ case the DOSs of the amorphous and crystalline parts are both similar to the amorphous bulk phase, while in the classical case the crystalline core preserves the DOS of the bulk crystalline phase. This confirms that the phonons feel the interface between the amorphous and the crystalline phases not as a sharp interface, but rather like a broad boundary with a thickness of $2 \mathrm{~nm}$. This has been also observed in a previous study [35] in the case of superlattices with crystalline and amorphous phases, where the DOS in the crystalline phase becomes similar to the one of bulk crystalline silicon in a distance of almost $1 \mathrm{~nm}$ from the amorphous/crystalline theoretical interface.

\section{C. $c c$ as diameter-modulated silicon nanowires (DMCS)}

As showed in the previous subsection, when the amorphous fraction is adequate the TC is reduced, by a factor of roughly 6 in the case of the core/shell nanowires compared to those of pristine crystalline nanowires. In this last section we consider a simple model of nanowire which could be relevant for thermoelectricity. For this purpose the electronic properties should be preserved as much as possible while the thermal conductivity is reduced. We should mention at this point that one expects a preservation of the electronic conductivity as

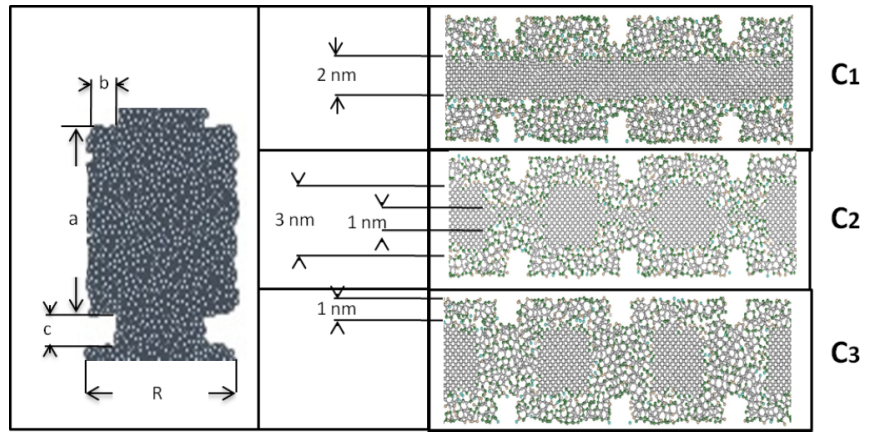

FIG. 10. (Color online) Snapshots of the three configurations used to model the diameter-modulated $c c / a s$ silicon nanowires. (a) $C 1$ configuration, with constant diameter crystalline core and diameter-modulated amorphous shell. (b) $C 2$ configuration, where both crystalline and amorphous regions are diameter modulated. (c) C3 configuration consisting of crystalline nanoinclusions inside a diameter-modulated amorphous shell.

the electron mean free path is much smaller than the one of the phonons $[48,49]$. For example, at room temperature and for electron doping of $\sim 10^{19} \mathrm{~cm}^{-3}$, averaged mean-free-path values can be extracted from transport measurements [50,51] and are of the order of $1-10 \mathrm{~nm}$ for electrons and $40 \mathrm{~nm}$ for phonons. However in a recent paper by B. Qiu and Tian [52] it was shown that the differences could be much larger for the electron and the phonon modes contributing to the conductivities. This leads to the conclusion that the electrons should feel much less the structural modulations compared to the phonons. Of course one should calculate the electronic conductivity of these nanostructures, but this is not in the scope of our paper. We investigate now the diametermodulated nanowires with crystalline core and amorphous shell $(c c / a s)$. The three configurations we consider are (see Fig. 10) (i) the $C 1$ configuration in which there is a continuous and constant diameter crystalline core with an amorphous modulated shell; (ii) the $C 2$ configuration in which both the crystalline core and the amorphous shell regions are diameter modulated and for which there is a continuous crystalline axis in the center of the nanowire; and (iii) the $C 3$ configuration in which there are crystalline nanoinclusions inside a diameter-modulated amorphous envelope. The TC of these three diameter-modulated core/shell nanowires is given in Fig. 11, and to achieve comparisons we added the results of the pristine crystalline and pristine amorphous diameter-modulated nanowires with the same size as the $C 1$, $C 2$, and $C 3$ configurations. To add to the discussion previously mentioned about the electronic conductivity, we are quite confident that in the case of the $C 1$ configuration, as there is no additional roughness at the interface between crystalline and amorphous phases, the electronic conductivity should be equal to the one of a pristine crystalline nanowire with diameter equal to the core diameter [53]. An additional roughness like in cases of the configurations $C 2$ and $C 3$ would deteriorate the electronic conductivity, and finally we cannot give a clear statement for the benefit to the overall figure of merit.

In Fig. 10 we observe that along the series $C_{1}, C_{2}$, and $C_{3}$ the central crystalline channel is being enclosed by the amorphous phase. The results for the thermal conductivity presented in 


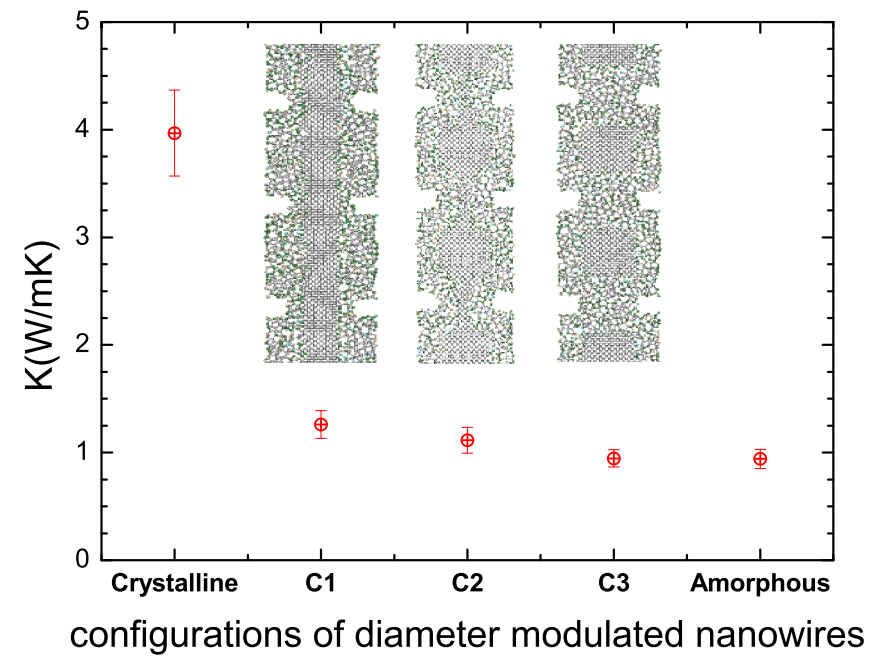

FIG. 11. (Color online) TC of the three diameter and phase modulated nanowires (DMCS) as presented in Fig. 10, compared with the pristine crystalline and pristine amorphous diameter-modulated nanowires with the same dimensions.

Fig. 11 show that it is reduced further and further along the series. The values for the $C_{2}$ and $C_{3}$ configurations are in fact very close to the one of the modulated nanowires with only amorphous phase. In the case of thermoelectricity we believe that $C_{2}$ is the most favorable one, since electronic transport should be preserved more compared to the $C 3$ configuration. In fact those results are complementary to the ones discussed in a previous work by He and Galli [16]. They studied DMCS nanowires with a larger diameter $(15 \mathrm{~nm}$ instead of our $5 \mathrm{~nm}$ ), but much shorter. They found that the TC for DMCS $a-S i / c-S i$ nanowires equals to $25 \%$ of the constant section crystalline nanowire. Here, we found a TC for the $C_{2}$ configuration equal to $17 \%$ of the crystalline constant section nanowire.

\section{CONCLUSION}

Here we presented models to lower, as low as the amorphous limit, the TC of silicon nanowires. The combined incorporation of the amorphous phase and the diameter modulations is a way to decrease the TC, without totally suppressing the electronic conductivity. Therefore, such design could increase the thermoelectric figure of merit of the nanowire. The $a c / c s$ nanowires are very interesting candidates for such purpose as they exhibit TC of half of the usual core/shell nanowires, and without diameter modulation, which is experimentally difficult to achieve. The addition of modulations and amor-

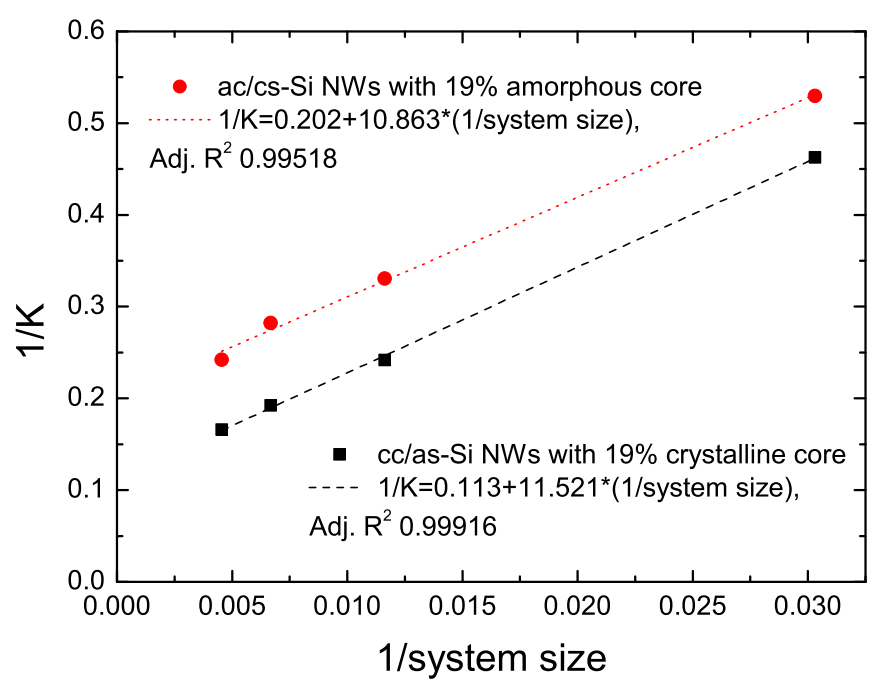

FIG. 12. (Color online) Inverse of the thermal conductivity vs the inverse system size.

phous/crystalline structures can lead to a TC close to the one of the pristine amorphous nanowire, while preserving a small portion of crystalline material in the core of the nanowire. The latter issue could be crucial for the transport of electrons. Further investigation is however necessary to confirm this statement.

\section{ACKNOWLEDGMENTS}

The authors are grateful for the discussions with Tristan Albaret of the Institut Lumière Matière of Lyon, Julien Guénolé of the University of Poitiers, and Vladimir Lysenko of the Institute of Nanotechnologies of Lyon, who communicated with us his interesting fabrication results. This work was performed using high-performance computing resources from GENCI-IDRIS (Grant No. 2014-x2014097186).

\section{APPENDIX}

In Fig. 12 the inverse of the thermal conductivity as a function of the inverse system size in the direction of the heat flow is given. The two types of core/shell NWs are given; the $c c / a s$ and $a c / c s$ nanowires are both with the same percentage of amorphous phase. In the inset the extrapolation equation and the statistical error of this extrapolation are given. The $R$ square (square of the correlation between the response values and the predicted response values) measures how successful the fit is, in explaining the variation of the data.
[1] N. Papanikolaou, J. Phys. Condens. Matter 20, 135201 (2008).

[2] C. Dames and G. Chen, J. Appl. Phys. 95, 682 (2004).

[3] D. Donadio and G. Galli, Nano Lett. 10, 847 (2010).

[4] A. 1. Boukai, Y. Bunimovich, J. Tahir-Kheli, J.-K. Yu, W. A. Goddard III, and J. R. Heath, Nature (London) 451, 168 (2008).

[5] A. 1. Hochbaum, R. Chen, R. D. Delgado, W. Liang, E. C. Garnett, M. Najaran, A. Majumdar, and P. Yang, Nature (London) 451, 163 (2008).
[6] K. Termentzidis, T. Barreteau, Y. Ni, S. Merabia, X. Zianni, Y. Chalopin, P. Chantrenne, and S. Volz, Phys. Rev. B 87, 125410 (2013).

[7] X. Zianni, V. Jean, K. Termentzidis, and D. Lacroix, Nanotechnology 25, 465402 (2014).

[8] X. Zianni, Appl. Phys. Lett. 97, 233106 (2010).

[9] X. Zianni, Nanoscale Research Letters 6, 286 (2011). 
[10] D. L. Nika, A. I. Cocemasov, D. V. Crismari, and A. A. Balandin, Appl. Phys. Lett. 102, 213109 (2013).

[11] D. L. Nika, A. I. Cocemasov, C. I. Isacova, A. A. Balandin, V. M. Fomin, and O. G. Schmidt, Phys. Rev. B 85, 205439 (2012).

[12] S. Crawford, S. K. Lim, and S. Gradeak, Nano Lett. 13, 226 (2013).

[13] G. D. Sulka, A. Brzózka, and L. Liu, Electrochim. Acta 56, 4972 (2011).

[14] D. Donadio and G. Galli, Phys. Rev. Lett. 102, 195901 (2009).

[15] F. Sansoz, Nano Lett. 11, 5378 (2011).

[16] Y. He and G. Galli, Phys. Rev. Lett. 108, 215901 (2012).

[17] W. Lu and J. Xiang, Semiconductor Nanowires, From NextGeneration Electronics to Sustainable Energy (Royal Society of Chemistry, Cambridge, 2015).

[18] P. J. Newby, B. Canut, J.-M. Bluet, S. Gomès, M. Isaiev, R. Burbelo, K. Termentzidis, P. Chantrenne, L. G. Frechette, and V. Lysenko, J. Appl. Phys. 114, 014903 (2013).

[19] V. Lysenko (private communication).

[20] C. A. da Cruz, K. Termentzidis, P. Chantrenne, and X. Kleber, J. Appl. Phys. 110, 034309 (2011).

[21] K.Termentzidis and S. Merabia, Molecular Dynamics: Theoretical Developments and Applications in Nanotechnology and Energy (InTech, Kroatia, 2012).

[22] K. Termentzidis, J. Parasuraman, C. Da Cruz, S. Merabia, D. Angelescu, F. Marty, T. Bourouina, X. Kleber, P. Chantrenne, and P. Basset, Nanoscale Research Letters 6, 288 (2011).

[23] P. K. Schelling, S. R. Phillpot, and P. Keblinski, Phys. Rev. B 65, 144306 (2002).

[24] K. Termentzidis, P. Chantrenne, and P. Keblinski, Phys. Rev. B 79, 214307 (2009).

[25] K. Termentzidis, S. Merabia, P. Chantrenne, and P. Keblinski, Int. J. Heat Mass Transf. 54, 2014 (2011).

[26] P. Chantrenne and J.-L. Barrat, Superlattices Microstruct. 35, 173 (2004).

[27] P. Chantrenne and J.-L. Barrat, J. Heat Transfer 126, 577 (2004).

[28] P. Keblinski, M. Z. Bazant, R. K. Dash, and M. M. Treacy, Phys. Rev. B 66, 064104 (2002).

[29] S. Plimpton, J. Comput. Phys. 117, 1 (1995).

[30] S. Plimpton, R. Pollock, and M. Stevens, in Proceedings of the Eighth SIAM Conference on Parallel Processing for Scientific Computing, 1997 (unpublished).
[31] F. H. Stillinger and T. A. Weber, Phys. Rev. B 31, 5262 (1985).

[32] R. Vink, G. Barkema, W. van der Weg, and N. Mousseau, J. Non-Cryst. Solids 282, 248 (2001).

[33] K. Termentzidis, A. France-Lanord, E. Blandre, T. Albaret, S. Merabia, V. Jean, and D. Lacroix, MRS Proceedings 1543, 71 (2013).

[34] A. France-Lanord, E. Blandre, T. Albaret, S. Merabia, D. Lacroix, and K. Termentzidis, J. Phys. Condens. Matter 26 , 055011 (2014).

[35] A. France-Lanord, S. Merabia, T. Albaret, D. Lacroix, and K. Termentzidis, J. Phys. Condens. Matter 26, 355801 (2014).

[36] S. Nose, J. Chem. Phys. 81, 511 (1984).

[37] K. Laaziri, S. Kycia, S. Roorda, M. Chicoine, J. L. Robertson, J. Wang, and S. C. Moss, Phys. Rev. Lett. 82, 3460 (1999).

[38] J. Fortner and J. S. Lannin, Phys. Rev. B 39, 5527 (1989).

[39] N. Bernstein, M. J. Aziz, and E. Kaxiras, Phys. Rev. B 58, 4579 (1998).

[40] C. M. Yang, Ph.D. thesis, California Institute of Technology, 1997.

[41] D. Li, Y. Wu, P. Kim, L. Shi, P. Yang, and A. Majumdar, Appl. Phys. Lett. 83, 2934 (2003).

[42] A. Rajabpour, S. M. V. Allaei, and F. Kowsary, Appl. Phys. Lett. 99, 051917 (2011).

[43] G. Domingues, J.-B. Saulnier, and S. Volz, Superlattices Microstruct. 35, 227 (2004).

[44] W. Humphrey, A. Dalke, and K. Schulten, J. Molec. Graphics 14, 33 (1996).

[45] H. K. Liu, Y. Lin, and S. N. Luo, J. Phys. Chem. C 118, 24797 (2014).

[46] T. Matsunaga, N. Yamada, R. Kojima, S. Shamoto, M. Sato, H. Tanida, T. Uruga, S. Kohara, M. Takata, P. Zalden et al., Adv. Funct. Mater. 21, 2232 (2011).

[47] B. Qiu, L. Sun, and X. Ruan, Phys. Rev. B 83, 035312 (2011).

[48] T. Feng and X. Ruan, Journal of Nanomaterials, 2014, 206370 (2014).

[49] S.-H. Ko and C. Grigoropoulos, Hierarchical Nanostructures for Energy Devices (Royal Society of Chemistry, UK, 2014).

[50] L. Weber and E. Gmelin, Appl. Phys. A 53, 136 (1991).

[51] J. S. Blakemore, Solid State Physics, 2nd ed. (Cambridge University Press, Cambridge, 1987).

[52] B. Qiu and Z. Tian, arXiv:1409.4862.

[53] P. N. Martin, Z. Aksamija, E. Pop, and U. Ravaioli, Nano Lett. 10, 1120 (2010). 